\title{
Editorial
}

\section{\#InSituPathologists: how the \#USCAP2015 meeting went viral on Twitter and founded the social media movement for the United States and Canadian Academy of Pathology}

David Cohen ${ }^{1}$, Timothy Craig Allen ${ }^{2}$, Serdar Balci ${ }^{3}$, Philip T Cagle ${ }^{1}$, Julie Teruya-Feldstein ${ }^{4}$, Samson W Fine ${ }^{5}$, Dibson D Gondim ${ }^{6}$, Jennifer L Hunt ${ }^{7}$, Jack Jacob ${ }^{8}$, Kimberly Jewett ${ }^{9}$, Xiaoyin 'Sara' Jiang ${ }^{10}$, Keith J Kaplan ${ }^{11}$, Ibrahim Kulac ${ }^{12}$, Rashna Meunier ${ }^{13}$, Nicole D Riddle ${ }^{14}$, Patrick S Rush ${ }^{15}$, Jennifer Stall ${ }^{16}$, Lauren N Stuart ${ }^{17}$, David Terrano ${ }^{18}$, Ed Uthman ${ }^{19}$, Matthew J Wasco ${ }^{20}$, Sean R Williamson ${ }^{21}$, Roseann I Wu ${ }^{22}$ and Jerad M Gardner ${ }^{7}$

${ }^{1}$ Department of Pathology and Genomic Medicine, Houston Methodist Hospital, Houston, TX, USA; ${ }^{2}$ Department of Pathology, The University of Texas Medical Branch, Galveston, TX, USA; ${ }^{3}$ Department of Pathology, Yildirim Beyazit University Faculty of Medicine, Ankara, Turkey; ${ }^{4}$ Department of Pathology, Icahn School of Medicine, Mount Sinai Health System, New York, NY, USA; ${ }^{5}$ Department of Pathology, Memorial Sloan Kettering Cancer Center, New York, NY, USA; ${ }^{6}$ Department of Pathology, Indiana University School of Medicine, Indianapolis, IN, USA; ${ }^{7}$ Department of Pathology, University of Arkansas for Medical Sciences, Little Rock, AR, USA; ${ }^{8}$ Department of Pathology, Montefiore Medical Center, Bronx, NY, USA; ${ }^{9}$ Kimberly Jewett Consulting, Inc., Plainfield, IL, USA; ${ }^{10}$ Department of Pathology, Duke University, Durham, NC, USA; ${ }^{11}$ Publisher, tissuepathology.com, Charlotte, NC, USA; ${ }^{12}$ Department of Pathology, Johns Hopkins University, Baltimore, MD, USA; ${ }^{13}$ Adirondack Pathology, Glens Falls, NY, USA; ${ }^{14}$ Cunningham Pathology (An Aurora Dx Partner), Birmingham, AL, USA ${ }^{15}$ Department of Pathology, University of Wisconsin Hospital \& Clinics, Madison, WI, USA; ${ }^{16}$ Hospital Pathology Associates, Minneapolis, MN, USA; ${ }^{17}$ Treasure Coast Pathology, Stuart, FL, USA; ${ }^{18}$ Departments of Pathology and Dermatology, Mount Sinai Hospital, New York, NY, USA; ${ }^{19}$ OakBend Medical Center \& PATHO-L, Richmond, TX, USA; ${ }^{20} I H A$ Pathology and Laboratory Medicine and St Joseph Mercy Hospital, Ann Arbor, MI, USA; ${ }^{21}$ Department of Pathology, Henry Ford Health System, Detroit, MI, USA and ${ }^{22}$ Department of Pathology and Laboratory Medicine, University of Pennsylvania Health System, Philadelphia, PA, USA

Professional medical conferences over the past five years have seen an enormous increase in the use of Twitter in real-time, also known as "live-tweeting". At the United States and Canadian Academy of Pathology (USCAP) 2015 annual meeting, 24 attendees (the authors) volunteered to participate in a live-tweet group, the \#InSituPathologists. This group, along with other attendees, kept the world updated via Twitter about the happenings at the annual meeting. There were 6,524 \#USCAP2015 tweets made by 662 individual Twitter users; these generated 5,869,323 unique impressions (potential tweet-views) over a 13-day time span encompassing the dates of the annual meeting. Herein we document the successful implementation of the first official USCAP annual meeting live-tweet group, including the pros/cons of live-tweeting and other experiences of the original \#InSituPathologists group members. No prior peer-reviewed publications to our knowledge have described in depth the use of an organized group to "live-tweet" a pathology meeting. We believe our group to be the first of its kind in the field of pathology.

Modern Pathology (2017) 30, 160-168; doi:10.1038/modpathol.2016.223; published online 13 January 2017

Correspondence: Assistant Professor JM Gardner, MD, Department of Pathology, University of Arkansas for Medical Sciences, 4301 W. Markham St. \#517, Little Rock, AR 72205, USA. E-mail: JMGardnerMD@gmail.com; Twitter: @JMGardnerMD Published online 13 January 2017
Social media has proven itself as a disruptive influence in how people communicate and disseminate information. Rather than waiting for information to permeate through traditional channels, social 
networks serve as a force amplifier with real-time communication linking individuals to data as soon as it is generated. Once limited to the personal realm, social media and social networking have quickly developed utility as a tool for physician learning and interaction.

Twitter, a popular social network microblogging platform introduced in 2006, allows users to rapidly send short 140 character 'tweets' or messages. Importantly, these 'tweets' can also include pictures, videos, internet links, and mentions of other Twitter users. Users can also tag messages using 'hashtags', a form of searchable and clickable labels, denoted by the \# symbol. Hashtags allow Twitter users to easily find tweets of interest.

Using a free application easily accessed on smartphones or computer, Twitter users can quickly send tweets to their followers who in turn can retweet (share a tweet with their own followers), reply (respond to the original tweet and initiate a discussion), like, or mention a particular interesting posting. ${ }^{1}$ As tweets are by default public, dedicated Twitter users can quickly develop a large following and reach a large number of individuals with even a single message.

As a highly visual specialty, pathology is especially conducive to this form of asynchronous communication. An image of a particularly interesting case or a presentation summary can rapidly be sent to followers and discussed with colleagues. Literally at their fingertips, most pathologists also have the ability to easily take a high-quality picture, a feature of most smartphones, through their microscope eyepieces, and then share de-identified images via social media stimulating instantaneous discussion and education within a potentially worldwide forum. ${ }^{2,3}$

Professional medical conferences over the past 5 years have seen an enormous increase in the use of Twitter in real time, also known as 'live-tweeting'. ${ }^{4-7}$ The primary focus of pathology conferences includes acquiring the most up-to-date research and conversing with colleagues. Rather than supplant these activities, including networking, which normally occurs at pathology meetings, Twitter enhances them by playing a complementary role in the rapid dissemination of information. Tweets, with conference-specific hashtags, allow research findings from the meeting to quickly expand far beyond the walls of the convention center. Tweets during meetings also help other attendees keep up with what they missed in a session they were unable to attend.

Perhaps most importantly, Twitter allows pathologists to carry on conversations long after a conference has ended. Anecdotally, we found that many relationships developed by interactions between pathologists on Twitter convert naturally into 'reallife' socialization and networking. ${ }^{8}$ In essence, Twitter provides a free easily accessible medium for the dissemination of new clinically relevant information to a worldwide audience beyond the confines of a conference or traditional subspecialty journals.

Although pathologists are increasingly utilizing social media, no prior peer-reviewed publications to our knowledge have described in depth the use of an organized group to 'live-tweet' a pathology meeting. ${ }^{9-13}$ We believe our group to be the first of its kind in the field of pathology. Here we document the successful implementation of the first official United States and Canadian Academy of Pathology (USCAP) annual meeting live-tweet group (referred to as the '\#InSituPathologists') at the 2015 USCAP annual meeting and its continuing success in $2016 .{ }^{14}$

\section{Material and methods}

\section{Formation of the Live-Tweet Group}

On February 16, 2015, one of the authors (JMG) tweeted a 'call to action' among regular pathologist users of Twitter asking for volunteers who were interested in live-tweeting the upcoming USCAP 2015 annual meeting (March 21-27, 2015; Boston, Massachusetts, USA) (Figure 1). ${ }^{15}$ This invitation was greeted with enthusiastic approval, and a group of Twitter users was formed. Dr Gardner then contacted the Executive Vice President of USCAP, Dr David Kaminsky, explaining the concept and requesting formal approval of the group. The Academy embraced the idea and formally supported the group. Dr Gardner polled group members for a potential name for this live-tweet group. Dr Samson Fine suggested (and a majority vote approved) the hashtag/name \#InSituPathologists.

\section{Twitter Quantitative Analysis and Survey Metrics}

Symplur.com, a healthcare-based Twitter metrics website, was used to analyze tweets and impressions (potential tweet views) that contained the registered \#USCAP2015 hashtag. This website tracks Twitterrelated activities centered on hashtags as part of the Healthcare Hashtag Project. ${ }^{16}$ Information concerning the number of mentions, tweets, and impressions

\section{(大) Jerad Gardner, MD Q.JMGardnerMD}

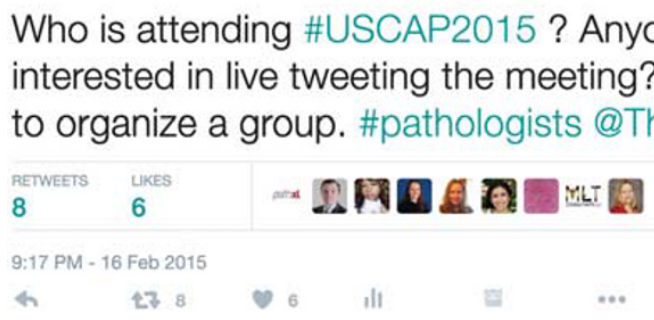

Figure 1 Initial 'call to action' tweet for the \#USCAP2015 livetweet group. This twitter message serves as an example of the use of hashtags (\#USCAP2015), tagging/mentions of other users (@TheUSCAP), and retweeting within a 140 character message. ${ }^{15}$ 
Table 1 Survey questions for members of the \#USCAP2015 \#InSituPathologists live-tweet group

1. When did you join Twitter?

2. What things did you enjoy about live-tweeting \#USCAP2015?

3. Any specific negatives or drawbacks?

4. Overall, was it a positive experience?

5 . Would you do it again?

6. Any specific advice, dos/don'ts, or pro tips for new Twitter users who want to live-tweet a pathology meeting?

7. Did you receive any feedback (either online or in real life) about our live-tweets from people who did not attend \#USCAP2015? If yes, please share examples.

during the conference period was retrospectively reviewed.

All members of the official \#InSituPathologists group were subsequently contacted to voluntarily answer 7 free-text survey questions (Table 1) regarding their experience live-tweeting at USCAP 2015 to provide feedback.

\section{Results}

\section{Formation and Demographics of the USCAP 2015 Live- Tweet Group}

Twenty-four volunteer attendees participated in the USCAP 2015 \#InSituPathologists live-tweet group. Members wore Twitter committee badge ribbons on their identification tags and agreed to 'live-tweet' during the conference using the approved \#USCAP2015 hashtag. With the help of Ethan Kaminsky (founder of Kaminsky Productions and marketing and advertising consultant for USCAP), a poster (Figure 2) was created showing the members of the live-tweet group and their credentials. This poster was shared on Twitter ahead of the meeting and was prominently displayed at the USCAP meeting to encourage participants to follow live-tweets using the conference specific \#USCAP2015 hashtag.

The 24-member live-tweet group was composed of 11 academic pathologists, 3 private practice pathologists, 8 pathology trainees, 1 senior medical student pursuing pathology residency, and 1 breast cancer survivor and patient advocate. In addition to general surgical pathology, group members represented the following pathology subspecialties: bone and soft tissue pathology, cytopathology, dermatopathology, genitourinary pathology, gynecologic pathology, head and neck pathology, hematopathology, molecular pathology, and pulmonary pathology. All members were in practice or training in the United States except for one pathologist practicing in Turkey. The following states were represented in the group: Arkansas (2), Georgia (1), Illinois (1), Indiana (1), Maryland (1), Massachusetts (1), Michigan (2), New York (4), North Carolina (2), Pennsylvania (1), Rhode Island (1), Texas (5), and Wisconsin (1). The members had been Twitter users for an average of 3 years (range: < 1-6 years). Age was not asked in the survey, but one member of the group included this comment voluntarily: 'I think I am the oldest InSituPathologist by far, so if you want to mention my age (63), that may encourage other (pathologists in this age range) to climb on the Twitter bandwagon.'

\section{Quantitative Analysis Results}

Using analytic data metrics from the healthcare metric site Symplur.com, 6524 \#USCAP2015 tweets created 5869323 unique impressions over a 13-day time span encompassing the dates of the annual meeting (Figure 3). A total of 662 individual Twitter users posted tweets using the conference hashtag; $68 \%$ of postings included a retweet. The top 10 \#USCAP2015 utilizers posted a combined 2840 tweets with 3529464 impressions $(60.4 \%$ of the total impressions). ${ }^{17}$ The members of the \#InSituPathologists livetweet group contributed 2199 tweets, accounting for $33.9 \%$ of the total tweets about \#USCAP2015. Symplur.com tracks potential impressions (the number of times tweets were delivered to the Twitter streams of other users and potentially viewed) rather than actual impressions (the number of times tweets were actually seen by other users); throughout this article, the term 'impressions' refers to potential impressions rather than actual impressions.

The greatest number of tweets was seen on the first day of the conference (1746 tweets) (Figure 4). Throughout the actual dates of the meeting (21-27 March 2015), the average rate of tweeting about \#USCAP2015 overall (not just limited to the livetweet group) was 36 tweets per hour with an average of 10 tweets per user. $^{18}$ The dissemination of \#USCAP2015-tagged tweets continued past the conference with an additional 566246 unique impressions and 909 tweets using the \#USCAP2015 hashtag occurring in the days immediately after the meeting ended. Tweets from the 2015 annual meeting are still being occasionally retweeted, having a potential impact even 17 months after the end of the meeting. As of the time of the preparation of this manuscript, the most recent \#USCAP2015 retweet (by someone other than one of the authors) was on 26 August 2016. ${ }^{19}$

\section{\#InSituPathologists Group Survey}

All 24 members of the \#InSituPathologists live-tweet group responded to the free text survey. Overwhelmingly, all participants described the experience as positive and found it worthwhile to pursue at future 


\section{(2)USCAP}

\section{"IN SITU PATHOLOGISTS" TWITTER GROUP MEMBERS}

\section{\#USCAP2015}

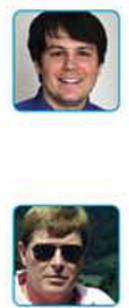

2) MGardnerMD

Jerad M. Gardner, M.D.

Assistant Professor of Pathology \& Dermatology

Dermatopathology Fellowship Program Director

University of Arkansas for Medical Sciences Little Rock, Arkansas

@TimAllenMDJD

Timothy Craig Allen, M.D., J.D.

Professor, Department of Pathology)

Director of Anatomic Pathology

The University of Texas Medical Branch Galveston, Texas

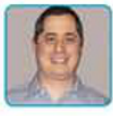

(a) Dibsong

Dibson D. Gondim, M.D.

Resident, Anatomic Pathology

Indiana University School of Medicine

Indianapolis, Indiana

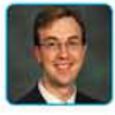

QGleason4plus5

Matthew Wasco, M.D.

Pathologist

IHA Pathology and Laboratory Medicine Ann Arbor, Michigan

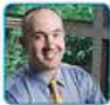

Qrovingatuscap

Samson W. Fine, M.D.

Associate Attending Pathologis

Memorial Sloan Kettering Cancer Center

New York, New York

Q9uzde54

Ibrahim Kulac, M.D.

Postdoctoral Fellow

Johns Hopkins University

Baltimore, Maryland

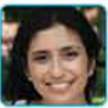

@RMeunierMD

Rashna Meunier, M.D.

Resident in Pathology, PGY-4

Brown University

Providence, Rhode Island

@feldstej

Julie Teruya-Feldstein, M.D.

Director of Hematopathology

Profeessor, Department of Pathology

Icahn School of Medicine,

Mount Sinai Health System

New York, New York

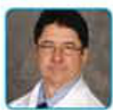

@PhilipTCagle

Philip T. Cagle, M.D.

Editor-in-Chief, Archives of Pathology \&

Laboratory Medicine

Directoc, Pulmonary Pathology, Department of Pathology and Genomic Medicine

Houston Methodist Hospital

Houston, Texas

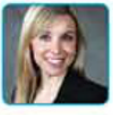

DrLaurenStuart

Lauren N. Stuart, M.D., M.B.A.

Chair, CAP Residents Forum

Pathology Resident, PGY-4

Emory University

Atlanta, Georgia

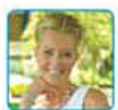

Q2XBCSURVIVOR76

Kimberly Jewett

$2 x$ Breast Cancer Survivor, Patient Advocate

CEO of Kimberty Jewett Consulting, Inc.

Plainfield, lllinois

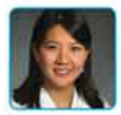

@ RoseannIWu

Roseann I. Wu, M.D., M.P.H.

Assistant Professor of Clinical Pathology

and Laboratory Medicine

University of Pennsylvania Health System

Philadelphia, Pennsylvania

Qeuthman

Ed Uthman, M.D.

Lab Director, OakBend Medical Center

Co-owner, PATHO-L

Richmond, Texas

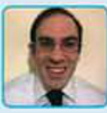

jackjacob

Jack Jacob

Medical Student, OMS IV

NYIT-College of Osteopathic Medicine Bronx, New York

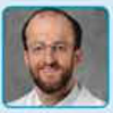

Williamson SR

Sean R. Williamson, M.D.

Clinical Assistant Profeessor of Pathology

Senior Staff Pathologist

Henry Ford Health System Detroit, Michigan

anRiddleMD

Nicole D. Riddle, M.D.

Assistant Professor of Pathology

Associate Residency Program Director

UT Health Science Center

San Antonio, Texas

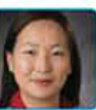

Sara_Jiang

Xiaoyin "Sara" Jiang, M.D.

Assistant Professor, Department of Pathology

Duke University

Durham, North Carolina

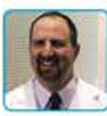

tissuepathology

Keith J. Kaplan, M.D.

Pathologist and Laboratory Medical Director

Publisher, tissuepathology.com

Charlotte, North Carolina

@jhuntpath

Jennifer Hunt, M.D.

Professor and Chair of Pathology

University of Arkansas for Medical Sciences Little Rock, Arkansas

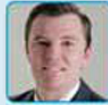

DrPatrickRush

Patrick S. Rush, D.0.

Pathology Resident, PGY2

University of Wisconsin Hospital \& Clinics

Madison, Wisconsin

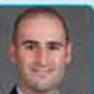

davidacohenmd

David Cohen, M.D.

Pathology Resident, PGY-2

Houston Methodist Hospital

Houston, Texas

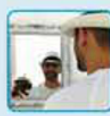

david_terrano

David Terrano, M.D., Ph.D.

Pathology Resident, PGY-4

New York Presbyterian Hospital, Columbia

University Medical Center

New York, New York

A2Path

Jennifer Stall, M.D.

Pobert E. Scully Fellow in Gynecologic

Pathology

Massachusetts General Hospital

Boston, Massachusetts

@serdarbalci

Serdar Balci, M.D.

Yildirim Beyazit University

Faculty of Medicin

Ankara, Turkey

Figure 2 The original 2015 \#InSituPathologists group. 
meetings. Although the responses varied, a few general themes were voiced among the majority of participants.
Rather than a static experience, live-tweeting felt interactive and participants enjoyed the real-time dynamic conversations. One respondent described it

\section{The \#USCAP2015 Influencers}

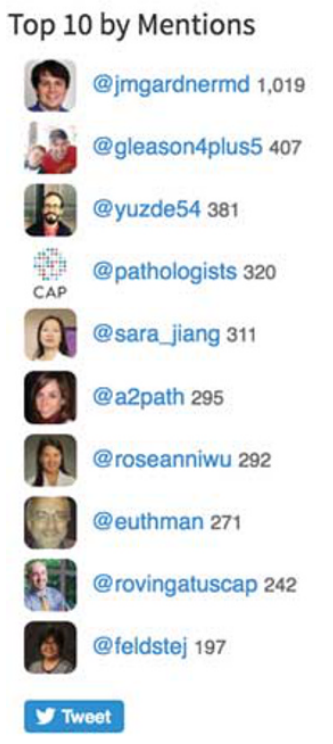

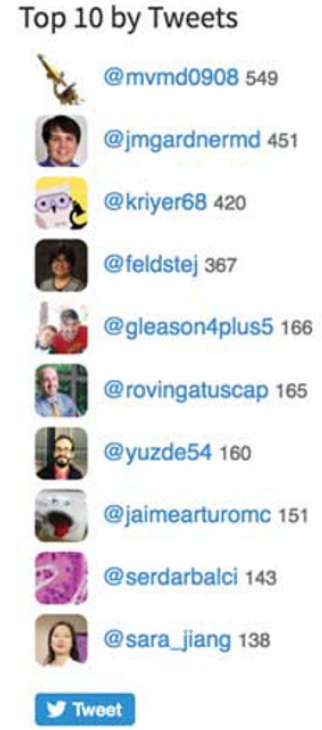

\section{The Numbers}

Top 10 by Impressions

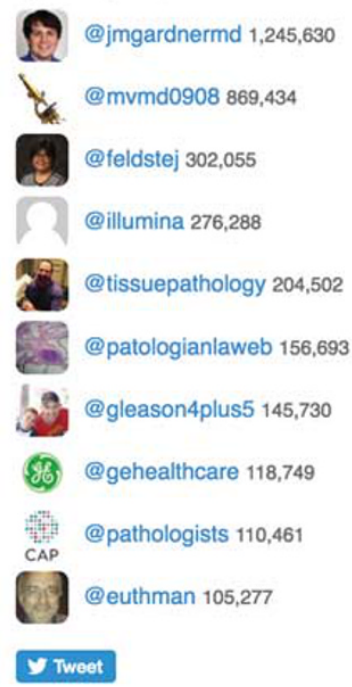

$5,869,323$ 6,524 nweets 662 particants 21 mermesteshour 10 mezinetespericionent

\#USCAP2015 analytics for time period 03/19/2015 00:00 to 04/01/2015 00:00 (Pacific Time: GMT-0700) [change time period]

Figure 3 Symplur.com Twitter activity metrics for \#USCAP2015 live-tweets including number of tweets and impressions as well as a ranking of users by number of mentions, tweets, and impressions generated. ${ }^{18}$

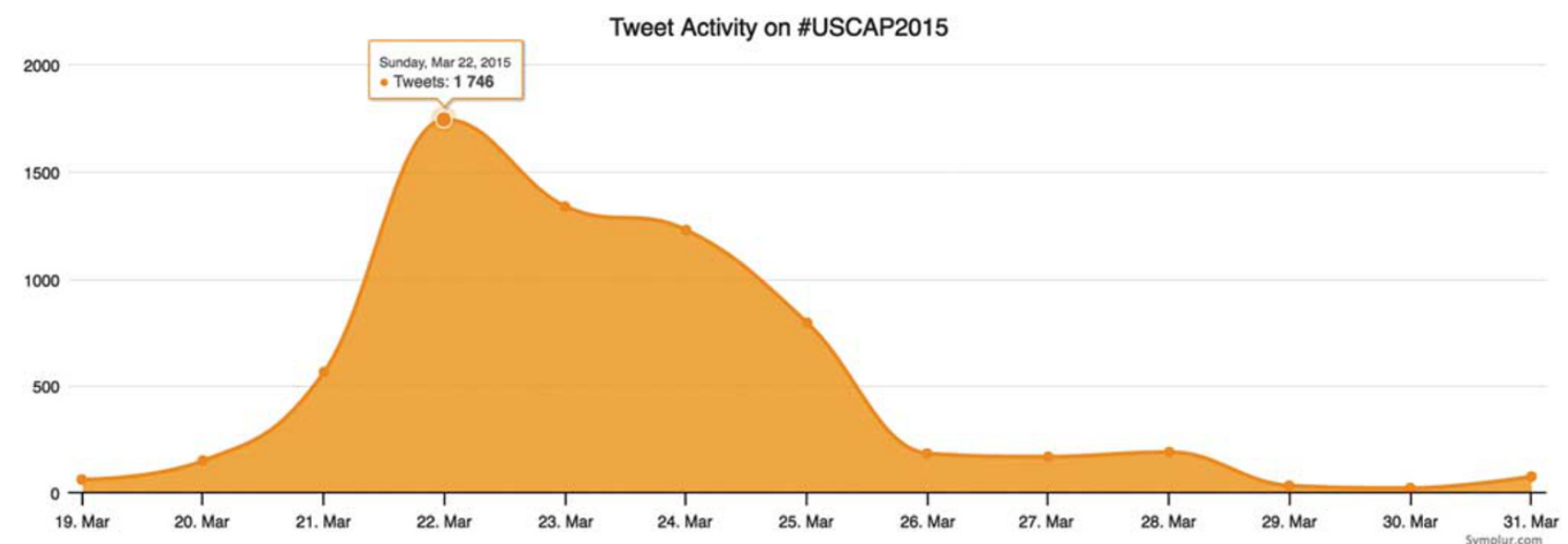

\#USCAP2015 Participants

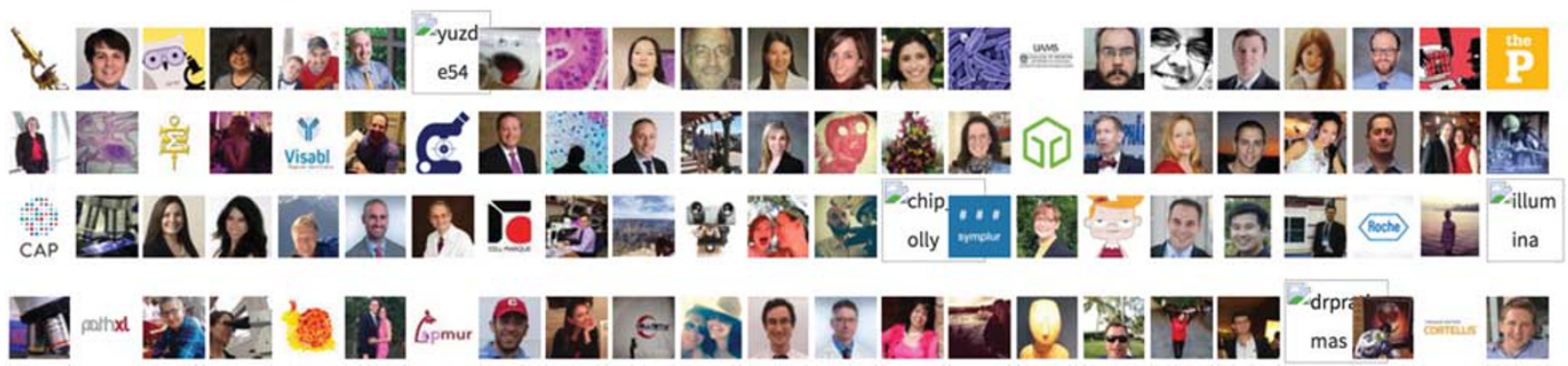

Figure 4 Number of \#USCAP2015 tweets ( $Y$ axis) graphed over time ( $X$ axis). The greatest number of tweets was on Sunday, the first day of the USCAP meeting. Graph courtesy of Symplur.com. 
as a 'more engaging experience' and likened it to driving a high-performance sports car. Many participants also noted the value of using Twitter as a personal educational adjunct. The ability to distill a conference topic into a short 140 character pearl or summary PowerPoint image was rewarding and a good way to condense lecture material into a more manageable learning experience. Another main benefit was being able to 'attend' multiple concurrent conferences simultaneously by following livetweets from concurrent lectures that would otherwise have been missed. Multiple participants mentioned camaraderie and networking as key advantages. Beyond the personal benefits provided by live-tweeting at the USCAP conference, participants felt rewarded in reaching a worldwide audience. In particular, there was recognition that many international pathologists and pathology trainees do not have the opportunity to directly attend USCAP. Through Twitter, these participants can enhance their own professional knowledge in a way that may not have been possible otherwise.

There were also a few negative elements to the live-tweet experience. One common criticism felt was the potential for distraction from the lecture attended when live-tweeting. Constant tweeting can also lead to a feeling of Twitter fatigue or burnout. Time spent on Twitter cannot only be a distraction during the lectures but can also detract from face-toface 'real-life' interaction with colleagues. Several members of the group were also concerned that obtrusively using their phone to tweet during a lecture might make them appear rude or inattentive to the speaker or to other members of the audience.

Members of the group received positive feedback from a variety of sources regarding the live-tweets from \#USCAP2015; comments came from individuals including pathology colleagues, international pathologists, physicians from other specialties (oncology, radiology, etc), patients and even nonmedical members of the public who were curious about what occurs at a pathology meeting.

Survey responses were compiled to produce a summary list of 10 'dos and do nots' for first-time users of Twitter at pathology conferences (Table 2).

\section{Implementation of Live-Tweeting for \#USCAP2016}

In preparation for the 2016 USCAP annual meeting, it quickly became apparent that there was an enormous increase in interest in Twitter among pathologists attending. The USCAP social media subcommittee decided to make an open invitation for the 2016 \#InSituPathologists group and instructed anyone wanting to be included to just tweet 'sign me up for \#InSituPathologists' and to tag the @TheUSCAP Twitter account. ${ }^{20}$ Badge ribbons were distributed at the meeting for live-tweet group participants. Approximately 275 different users utilized the \#InSituPathologists hashtag in the month leading up to the 2016 USCAP meeting. Although not a perfect measure, this suggests that somewhere in the range of 200-300 pathologists on Twitter indicated a willingness to live-tweet the \#USCAP2016 meeting. During the time period of the 2016 meeting, there were nearly 19000 tweets, made by over 1200 different users, which had the potential to be viewed over 28.5 million times (Figure 5).

\section{Discussion}

The use of meeting-specific Twitter hashtags to increase communication has rapidly grown in the last 5 years. A number of large clinical specialty annual meetings, including the American Society of Clinical Oncology, the American Urologic Association, the International Conference on Emergency Medicine, the American Society of Breast Surgeons, and the Academic Surgical Congress have documented the successful use of live-tweeting at their meetings. ${ }^{5,21-24}$ To the best of our knowledge, however, there has not yet been a peer-reviewed publication documenting in depth the organized live-tweeting of a pathology meeting and the use of a pathology conference-specific hashtag.

Although a small grassroots effort, the initial USCAP official live-tweet team and the \#USCAP2015 hashtag were a strong success with $>5$ million potential tweet-views generated. The endeavor was likely the largest formally organized pathology-related live-tweet group in history as of the date of the USCAP 2015 annual meeting. Using negligible resources aside from volunteered time, live-tweeting increased the learning opportunities for both pathologists at the conference and for those who could not attend USCAP both nationally and around the world. In addition, the use of the conference- and diagnosis-specific hashtags allowed informative, timely, and pertinent information from USCAP to reach our clinical colleagues in other specialties. $^{25}$

The use of an official organized live-tweet group served a number of purposes by ensuring a large proportion of the \#USCAP2015 tweets contained quality informative messages from trusted and vetted Twitter users $(33.9 \%$ of total tweets from the meeting were made by members of our group). Because of the diversity of the \#InSituPathologists group, including senior academic pathologists, younger subspecialty practicing pathologists, trainees, and a patient care advocate, a broader variety of opinions and topics of discussion were generated by our tweets, thus appealing to a potentially wider audience. Hashtag utilization metrics via Symplur.com, as well as individual Twitter user analytic data via Twitter Analytics, provided a wealth of free granular data about the use of Twitter at the annual meeting, which may serve a valuable purpose to attendees as well as future annual meeting organizers. 
Table 2 Ten dos and do nots: rules for live-tweeting at a pathology meeting

(1) Photos are golden

(2) Thoughts are good, pearls of wisdom are better

(3) Be respectful

(4) Use hashtags

(5) Find balance

(6) Do not be afraid to interact

(7) Stay transparent and professional

(8) Diversify the live- tweet process

(9) Avoid divisive or derogatory content

(10) Retweeting is your friend
Tweets with pictures get more interaction and avoid the limitations of 140 character messages. People will pay attention if the eye is drawn to something interesting.

Tweet intellectual nuggets or pearls that are not commonly known or that correct a common misconception. A thoughtful comment is better than an immediate play-by-play statement. Use a triad of factoids to enhance memorization.

Respect proprietary or embargoed information and give credit for content. While in session, be courteous to those around you in the audience both when typing and when taking pictures.

Use the meeting hashtag (eg-\#USCAP2016) and a specialty-specific hashtag (eg-\#pathology, \#dermpath, \#GIpath, \#GUpath, etc). This allows others interested in these topics to more easily find your tweet among all the rest. Pathology hashtag list: http://goo.gl/8iludP

Do not focus too much on your phone and miss the point or lessons of the talk. Do not tweet constantly; take the time to be present in the session to enjoy the meeting. Avoid burnout.

Do not be afraid to interface with other Twitter users. Reply to tweets with questions or comments to initiate conversations. Use the 'quote tweet' function to retweet excellent points made by others while adding your own comments and insights. Expressing a valuable point and/or providing expertise amplifies the quality of information.

Including a professional picture and academic/hospital affiliations in your Twitter profile adds credibility and background information to your content.

Tweeting in subspecialty conferences or individual companion pathology society meetings (ie-Arthur Purdy Stout Society) rather than just the plenary session is an opportunity for live-tweet users to provide valuable information on a conference that others may not be able to attend.

As with any social media platform, be aware that personal comments and non-professional opinions are publicly viewable. Even if you delete your tweet later, there is no way to ensure that someone else has not already saved or shared it. If you would not want your grandmother to read it, do not tweet it. Retweeting and including internet links or references helps disseminate useful postings. Other users will begin to value you as you are promoting their content and helping build their followers. In turn, retweeting helps you pick up more followers, too. Clicking the meeting hashtag or \#pathology are good starting points for finding tweets to retweet.
The \#USCAP2016 Influencers

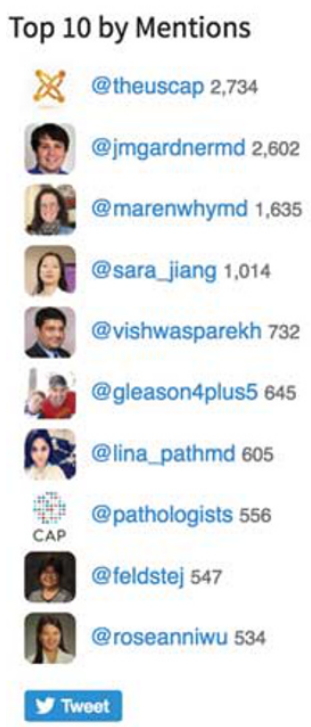

\section{Top 10 by Tweets}

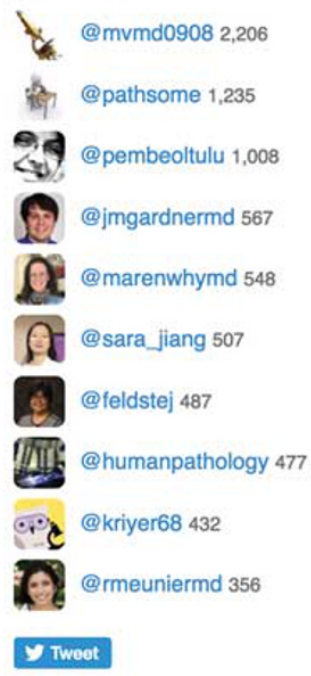

Top 10 by Impressions

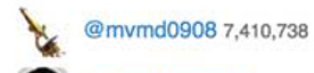

@ijmgardnermd 3,066,091

1] @if. @umanpathology 2,973,352

@thescientifik 1,700,427

Q8)

\& @ theuscap 933,193

e.

Q1 @sara_jiang 673,239

Q. @haneen_maghrabi 626,025

A. @ rmeuniermd 580,104
The Numbers
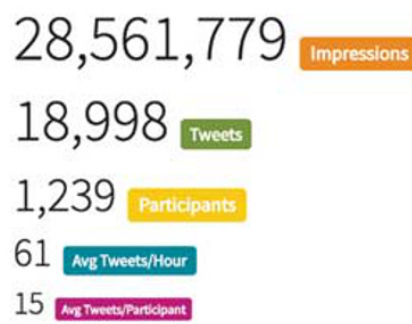

Tweet it Like

HUSCAP2016 analytics for time period 03/10/2016 00:00 to 03/23/2016 00:00 (Pacific Time: GMT-0700) [change time period]

Figure 5 Symplur.com Twitter activity metrics for \#USCAP2016 live-tweets including number of tweets and impressions as well as a ranking of users by number of mentions, tweets, and impressions generated. The 2016 USCAP meeting showed a drastic increase in Twitter activity compared with the 2015 annual meeting. ${ }^{26}$ Graph courtesy of Symplur.com.

In addition, the Twitter metrics provided powerful, tangible evidence of the success of the \#InSituPathologists live-tweet group. What started out as a grassroots social media experiment in
February 2015 resulted in over 6500 tweets about the \#USCAP2015 annual meeting at negligible financial cost to the organization. These metrics helped facilitate the subsequent formation of the 
USCAP Social Media Subcommittee chaired by one of the authors (JMG). ${ }^{14}$

The increase in Twitter activity between \#USCAP2015 and \#USCAP2016 was explosive. Just 1 year after our initial efforts, we saw $\sim 29$ million impressions generated at \#USCAP2016. In comparison with 2015, the 2016 annual meeting had double the number of Twitter users, triple the number of tweets, and quadruple the number of impressions. ${ }^{26}$ These levels of growth showcase the reach of Twitter and demonstrate its effectiveness when successfully applied to the field of pathology.

As an example, live-tweeting during the 2015 USCAP meeting afforded a real-time dissemination of the announcement and public reception of the terminology evolution from 'encapsulated follicular variant of papillary thyroid carcinoma' to the new nomenclature 'noninvasive follicular thyroid neoplasm with papillary-like nuclear features' (NIFTP), a major shift in the practice of thyroid cancer treatment that was first announced at \#USCAP2015. ${ }^{27}$ The announcement by the Endocrine Pathology Society was immediately tweeted by one of the original authors of the paper and by numerous other meeting attendees. ${ }^{28} \mathrm{~A}$ full year before the manuscript was published by Nikiforov et al and before The New York Times prominently described this paradigm shift to the lay public, NIFTP was already being actively discussed publicly on Twitter due to \#InSituPathologists who live-tweeted it from the USCAP 2015 annual meeting. ${ }^{29}$

\section{Summary}

In just 1 year from our original grassroots efforts to coordinate live-tweet coverage of the \#USCAP2015 annual meeting, the \#InSituPathologists live-tweeting concept has taken on a life of its own. All that was needed was an initial push and a dedicated group of pathologists to demonstrate the value and benefits of live-tweeting at pathology meetings. The authors believe that the initial \#InSituPathologists group played a critical role in providing that impetus, and we look forward to seeing continued growth and positive impact from live-tweeting at \#USCAP2017 and other pathology meetings far into the future.

\section{Disclosure/conflict of interest}

The authors declare no conflict of interest.

\section{References}

1 Thompson MA, Majhail NS, Wood WA, et al. Social media and the practicing hematologist: Twitter 101 for the busy healthcare provider. Curr Hematol Malig Rep 2015;10:405-412.

2 Morrison AO, Gardner JM. Microscopic image photography techniques of the past, present, and future. Arch Pathol Lab Med 2015;139:1558-1564.
3 Crane GM, Gardner JM. Pathology image-sharing on social media: recommendations for protecting privacy while motivating education. AMA J Ethics 2016;18: 817-825.

4 ASCO. ASCO 2015 will be the most social congress ever: creation knowledge, 2016. Available at http://creation.co/ knowledge/asco-2015-will-be-the-most-social-congress-ever/ (accessed 6 September 2016).

5 Attai DJ, Radford DM, Cowher MS. Tweeting the meeting: Twitter use at the American Society of Breast Surgeons Annual Meeting 2013-2016. Ann Surg Oncol 2016;23:3418-3422.

6 Attai DJ, Sedrak MS, Katz MS, et al. Social media in cancer care: highlights, challenges \& opportunities. Future Oncol 2016;12:1549-1552.

7 Chaudhry A, Glode LM, Gillman M, et al. Trends in twitter use by physicians at the American Society of Clinical Oncology Annual Meeting, 2010 and 2011. J Oncol Pract 2012;8:173-178.

8 Gardner JM, How to jumpstart your pathology career via social media: Youtube, 2016. Available at https:// www.youtube.com/watch? $\mathrm{v}=920 \mathrm{RUOzjEM} 4$ (accessed 6 September 2016).

9 Allen TC. Social media: pathologists' force multiplier. Arch Pathol Lab Med 2014;138:1000-1001.

10 Fuller MY, Allen TC. Let's have a Tweetup: the case for using twitter professionally. Arch Pathol Lab Med 2016;140:956-957.

11 Gardner JM. The social pathologist. The Pathologist 2015;5:18-19.

12 Glassy EF. The rise of the social pathologist: the importance of social media to pathology. Arch Pathol Lab Med 2010;134:1421-1423.

13 Misialek MJ, Allen TC. You're on social media! So now what? Arch Pathol Lab Med 2016;140:393.

14 Gardner JM. USCAP'S Social Media Committee rocks! USCAP Open Mind. 2016 Summer. http://www.uscap. org/public/newsletter/OpenMind_SUMMER_2016.pdf. (accessed 15 December 2016).

15 Gardner Jerad, Twitter post, 9:17 PM, 16 Feb 2015. Available at https://twitter.com/JMGardnerMD/status/ 567508180100325376.

16 Pinho-Costa L, Yakubu K, Hoedebecke K, et al. Healthcare hashtag index development: identifying global impact in social media. J Biomed Inform 2016;63: 390-399.

17 The \#USCAP2015 Influencers. \#USCAP2015 Analytics 2015 (cited 03/19/2015-04/01/2015). http://www.symplur. com/healthcare-hashtags/USCAP2015/analytics/?hashtag= USCAP2015\&fdate $=03 \% 2 \mathrm{~F} 19 \% 2 \mathrm{~F} 2015 \&$ shour $=00 \& \mathrm{smin}=$ 00\&tdate $=04 \% 2 \mathrm{~F} 01 \% 2 \mathrm{~F} 2015 \&$ thour $=00 \& \operatorname{tmin}=00$.

18 The \#USCAP2015 Influencers. \#USCAP2015 Analytics 2015, cited 03/21/2015-03/27/2015). http://www.symplur. com/healthcare-hashtags/USCAP2015/analytics/?hashtag= USCAP2015\&fdate $=03 \% 2 \mathrm{~F} 21 \% 2 \mathrm{~F} 2015 \&$ shour $=00 \& \mathrm{smin}=$ 00\&tdate $=03 \% 2 \mathrm{~F} 27 \% 2 \mathrm{~F} 2015 \&$ thour $=23 \& \operatorname{tmin}=59$.

19 \#USCAP2015 Conference Symplur, 2015. Available at http://www.symplur.com/healthcare-hashtags/uscap2015/.

20 USCAP (@USCAP), Twitter post, 7:11 AM, 2 March 2016. Available at https://twitter.com/theuscap/status/ 705002551373131777.

21 Cochran A, Kao LS, Gusani NJ, et al. Use of Twitter to document the 2013 Academic Surgical Congress. J Surg Res 2014;190:36-40.

22 Djuricich AM, Zee-Cheng JE. Live tweeting in medicine: 'Tweeting the meeting'. Int Rev Psychiatry 2015;27: 133-139. 
23 Logghe H, Maa J, Schwartz J. Twitter usage at Clinical Congress rises markedly over two years. Bull Am Coll Surg 2013;98:22-24.

24 Neill A, Cronin JJ, Brannigan D, et al. The impact of social media on a major international emergency medicine conference. Emerg Med J 2014;31:401-404.

25 Katz MS, Utengen A, Anderson PF, et al. Disease-specific hashtags for online communication about cancer care. JAMA Oncol 2016;2:392-394.

26 The \#USCAP2016 Influences: Symplur, 2016 (cited 03/10/2016-03/23/2016. http://www.symplur.com/health care-hashtags/USCAP2016/analytics/?hashtag=USCAP 2016\&fdate $=03 \% 2 \mathrm{~F} 10 \% 2 \mathrm{~F} 2016 \&$ shour $=00 \& \operatorname{smin}=00 \&$ tdate $=03 \% 2 \mathrm{~F} 23 \% 2 \mathrm{~F} 2016 \&$ thour $=00 \& \mathrm{tmin}=00$.

27 Nikiforov YE, Seethala RR, Tallini G, et al. Nomenclature revision for encapsulated follicular variant of papillary thyroid carcinoma: a paradigm shift to reduce overtreatment of indolent tumors. JAMA Oncol 2016;2: 1023-1029.

28 Sadow Peter, Twitter post. 9:42 PM, 21 Mar 2015 Available at https://twitter.com/PathDocBoston/status/ 579458086139781120.

29 Kolata G. It's Not Cancer: Doctors Reclassify a Thyroid Tumor. The New York Times, 2016. 